\title{
Towards Recognition of Control Variables for an Exoskeleton
}

\author{
Xanthi Papageorgiou, Joe McIntyre and Kostas J. Kyriakopoulos
}

\begin{abstract}
In this paper we present a methodology to drive the end effector of a robotic manipulator, to which is attached a human hand, in order to follow the human's intention of movement. This set-up is inspired from a Neuro-Robotics scenario in order to develop systems for restoring motor functionalities in injured and disabled people. Three typical tasks are considered, namely the robot not to interfere with the human's motion, to assist a person with limited motion capabilities, and finally to be used from the subjects for rehabilitation reasons. The proposed controllers utilize a force control in two different ways, with inner position loop and with inner velocity loop. The derived controllers attempt to incorporate neuroscientific models results. Also, stability and robust analysis is presented. The properties of the proposed methodology are verified through non-trivial computer simulations.
\end{abstract}

\section{INTRODUCTION}

In recent years, many scientific and technological efforts have been devoted to create Hybrid Bionic Systems (HBS) that link, via neural interfaces, the human nervous system with electronic and/or robotic artifacts. In general, this research has been carried out with various aims: on the one hand, to develop systems for restoring motor and sensory functionalities in injured and disabled people; on the other hand, for exploring the possibility of augmenting sensorymotor capabilities of humans in general, not only of disabled people. HBS can be generically defined as systems that contain both technical (artificial) and biological components. They can include:

- artificial systems with biological elements or subsystems. In such a case, the biological system is a complementary or supplementary element to the technical system

- biological systems with artificial elements or subsystems. The artificial subsystem, e.g. a robotic artefact, is a complementary or supplementary element to the biological system.

A possible scenario for an envisaged HBS is the "Beyond Orthesis", [1]. We define orthesis as devices which are externally attached to the human's upper limb for the support or for the improvement of its function. Some

This work is partially supported by the European Commission through contract "FP6 - IST - 001917 - NEUROBOTICS: The fusion of Neuroscience and Robotics".

$\mathrm{X}$. Papageorgiou is a $\mathrm{PhD}$ Student in the Mechanical Engineering Department, National Technical University of Athens, Greece, xpapagemail.ntua.gr

J. McIntyre is with the Laboratoire de Physiologie de la Perception et de l'Action, College de France, 75005 Paris, France, joe.mcintyre@college-de-France. fr

K.J. Kyriakopoulos is with the Faculty of Mechanical Engineering, National Technical University of Athens, Greece, kkyria@mail.ntua.gr

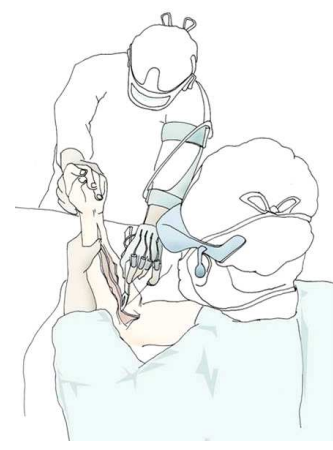

Fig. 1. Micro-surgery example, in which improvement of accuracy and endurance may be needed.

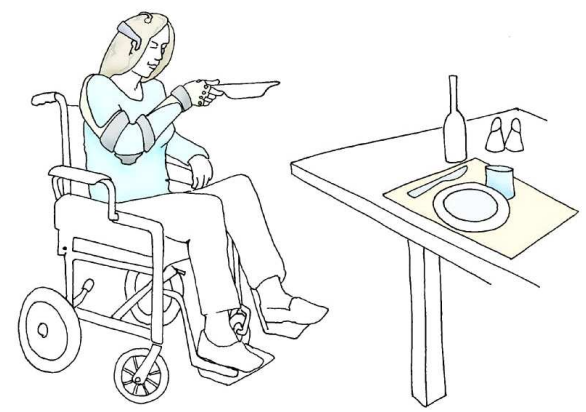

Fig. 2. Recovering human performance example, in which an intelligent exoskeleton is worn by a motor disabled person, in order to re-gain the use of the upper limb.

typical applications of "Beyond Orthesis" is an intelligent exoskeleton in a fine manipulation task, in order to improve human accuracy and endurance in micro-surgery operations (Fig. 1), or the recovering of human performance, in case of motor impairment (Fig. 2).

The use of an exoskeleton worn by a human subject requires a co-operative control scheme in which the intention of the movement must be detected by the mechatronic system and the motors must apply appropriate torque such that the exoskeleton aids, rather than inhibits, movement of the arm. A fundamental issue in the Neuro-Sciences related to this problem is that of identifying the control variables used by the central nervous system to control muscle activation and movements. Does the brain control force, torque, velocity or position, or does it control some other related variable? Does this suggest a means by which the interaction between the real arm and the exoskeleton should be carried out?

The main goal of this paper is to study the coupled system of human hand and an artificial arm (robotic manipulator). What we would like to implement is the robotic arm to 
recognize and assist the human's arm motion. We have performed an engineering analysis of how we can control a coupled system between a robotic manipulator and a human hand using information from a force/torque sensor attached between the artificial and the real hand. This analysis is based on some neuro-scientific approaches of how humans perceive and reproduce forces.

The rest of the paper is organized as follows: Section II formally states the considered problem, introducing preliminary definitions and notation, required for further discussion. Section III describes the appropriate analysis for force control while section IV presents the Equilibrium Position control. Section V presents the stability and robustness analysis for the proposed methodology. Section VI presents the simulation results and the paper concludes with section VII.

\section{PRELIMINARIES}

Our analysis is demonstrated by considering the requirement for a co-operative control scheme of an exoskeleton worn by a human subject (Fig. 3(a)). It is known that human subjects modulate stiffness according to the task at hand. According to this statement we will study a simplified setup in which the robotic hand is placed against the human subject's hand (Fig. 3(b)).

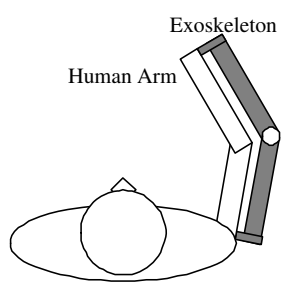

(a)

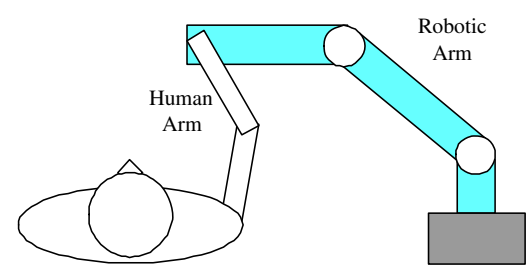

(b)
Fig. 3. (a) An exoskeleton set-up. (b) A simplified exoskeleton set-up.

In this simple scenario we will test how we can control the robotic manipulator. This manipulator is attached at the human's hand. The attempt to control the robot is based on the force information, a force/torque sensor can provide. This sensor is the only interface between the subject and the robot. We would like to recognize the human's intention of movement in order to drive the manipulator to execute the right movement.

All the possible scenarios that we will investigate in this work, are summarized in the following items. We would like the artificial arm to succeed in are the following distinguished tasks:

1) Reproduce the motion executed by the human, in order to achieve zero interaction force between human and robot. In other words, our scope is the robot not to interfere to the humans movement.

2) Amplify the force provided by the operator assisting the human hand during tasks. When small force is applied by the human subject, the robot can amplifies the force, in order to assist the human for example, to take a load (maybe for a person with motion disabilities).

3) Apply a specific force profile dependent on the force provided by the operator for rehabilitation of an injured person (exercise and training the motor activities of an injured person).

Furthermore, a basic goal of this work is to implement the above ideas by using the human's way of control the movements. For this reason we have based our analysis to some neuro-scientific models.

\section{A. Neuro-Scientific Models}

A number of hypotheses exist concerning how forces exerted by the arm on external objects are programmed by the Central Nervous System (CNS), [2]. Also, other studies showed that subjects measure a "sense of effort" associated with the muscle activity required to produce the perceived force, [3], [4]. A recent Neuro-Scientific study was concerned with the perception and reproduction of forces by the human hand, [5]. A main scope of this work was to point out which are the control variables used to perceive and reproduce forces by the humans. One of the conclusive results of [5] is that human subjects specify and store forces to be applied by the hand not in terms of a perceived force vector, but rather in terms of the motor activity required to resist or produce the force i.e., subjects possess a multi-dimensional "sense of effort".

Assuming that muscles and reflexes can be considered as tunable springs, a central motor command (effort) will define the equilibrium position $(E P)$ and impedance of the limb, [6], [7]. Experiments provided evidence that human subjects do not directly control forces or joint torques in a force reproduction task. Forces appear to be specified via control signals that modify the equilibrium position of the limb without regard for the impedance of the interaction with the environment. These results give credence to the idea that humans possess a "sense of effort" as opposed to a true "sense of force" when controlling forces in multiple dimensions.

Inspired of the above human's behavior model, we will try to implement and test this idea to the robotic manipulator. Firstly, we will apply a pure force control to the manipulator, according to the provided force from the human hand. Furthermore we will try to apply a control law to the robot arm based on the EP of the human arm.

\section{B. Robotic Manipulator}

We assume now that we have a stationary known environment and we consider a $m-d . o . f$. robot manipulator, which can be described trivially by a fully actuated second order dynamic model as the following:

$$
B(q) \cdot \ddot{q}+C(q, \dot{q}) \cdot \dot{q}+g(q)+J(q)^{T} \cdot f=\tau
$$

where $B(q)$ is the inertia matrix, $C(q, \dot{q})$ is the Coriolis term, $g(q)$ is the Gravity term, $q=\left[q_{1} \ldots q_{m}\right]^{T} \in \mathbb{R}^{m}$ is the vector of arm joint variables, $J(q)$ is the geometric Jacobian matrix, $f=\left[\begin{array}{ll}F^{T} & M^{T}\end{array}\right]^{T}$ denotes the vector 
of forces $F=\left[\begin{array}{lll}F_{x} & F_{y} & F_{z}\end{array}\right]^{T} \in \mathbb{R}^{3}$ and moments $M=$ $\left[\begin{array}{lll}M_{x} & M_{y} & M_{z}\end{array}\right]^{T} \in \mathbb{R}^{3}$ exerted by the end-effector of the robot manipulator on the environment, and $\tau \in \mathbb{R}^{m}$ the joint torque inputs, [8].

Using inverse dynamics control law with force measurement we have that:

$$
\tau=B(q) \cdot y+C(q, \dot{q}) \cdot \dot{q}+g(q)+J^{T}(q) \cdot f
$$

Substituting the control law from (2) to the system (1) we have that:

$$
\ddot{q}=y
$$

which describes the linearized equation of the system. The main goal now is to control the above linear system in order to achieve the predefined goals (control of the coupled system between human and artificial limb).

\section{FORCE CONTROL ANALYSIS}

What we would like to do is to control the robot manipulator using the interaction forces between the human hand and the robot manipulator. We have analyzed two cases of control law in order to control the measured force: force control with inner position loop and force control with velocity inner loop.

\section{A. Force Control with Inner Position Loop}

We have constructed a control law for the system described by (3) of the following equation:

$$
y=J_{A}^{-1}(q) \cdot M_{d}^{-1} \cdot\left(-K_{D} \cdot \dot{x}-K_{p} \cdot x-M_{d} \cdot \dot{J}_{A} \cdot \dot{q}+u\right)
$$

where $M_{d}$ is a positive definite mass matrix, $K_{D}$ is a positive definite damping matrix, $K_{p}$ is a positive semi-definite stiffness matrix, $J_{A}(q)$ is the analytic Jacobian matrix, $q=$ $\left[q_{1} \ldots q_{m}\right]^{T} \in \mathbb{R}^{m}$ is the vector of arm joint variables, $x=$ $\left[\begin{array}{ll}p^{T} & \phi^{T}\end{array}\right]^{T}$ is the vector of position $p=\left[\begin{array}{lll}x & y & z\end{array}\right]^{T} \in$ $\mathbb{R}^{3}$ and orientation $\phi=\left[\begin{array}{ccc}\varphi & \vartheta & \psi\end{array}\right]^{T} \in \mathbb{R}^{3}$ of the endeffector of the robot manipulator, and $u \in \mathbb{R}^{6}$ is the control input in the operational space. The corresponding block scheme is reported in Fig. 4.

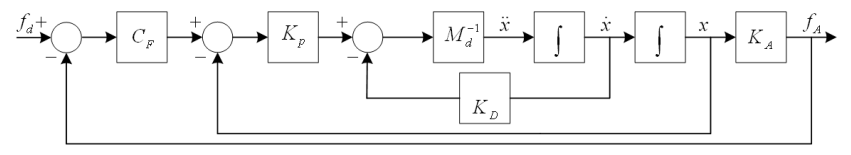

Fig. 4. Block diagram of force control with inner position loop.

Using the direct kinematics of the robot manipulator which are:

$$
x=k(q)
$$

we have that:

$$
\begin{gathered}
\dot{x}=\frac{\partial k(q)}{\partial q} \cdot \dot{q}=J_{A}(q) \cdot \dot{q} \\
\ddot{x}=J_{A}(q) \cdot \ddot{q}+\dot{J}_{A}(q) \cdot \dot{q}
\end{gathered}
$$

Substituting (4), (7) to the system (3) we have that:

$$
M_{d} \cdot \ddot{x}+K_{D} \cdot \dot{x}+K_{p} \cdot x=u
$$

which is the equation of the system in operational space.

\section{B. Force Control with Inner Velocity Loop}

We have constructed a control law for the system described by (3) of the following equation:

$$
y=J_{A}^{-1}(q) \cdot M_{d}^{-1} \cdot\left(-K_{D} \cdot \dot{x}-M_{d} \cdot \dot{J}_{A} \cdot \dot{q}+u\right)
$$

in the same notation with equation (4). The corresponding block scheme is reported in Fig. 5.

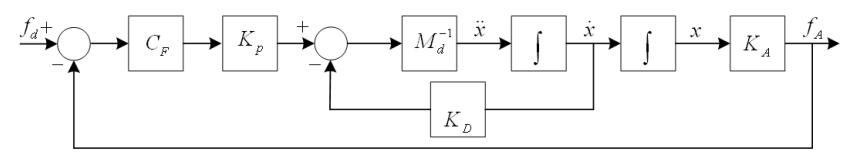

Fig. 5. Block diagram of force control with inner velocity loop.

Following the same procedure as from the previous case we have that the system (3) becames:

$$
M_{d} \cdot \ddot{x}+K_{D} \cdot \dot{x}=u
$$

The above equation describes the system in operational space. The main difference between (8) and (10) is that in the second approach, the force input affects directly the velocity of the systems which means that when the force provided by the human user is going to zero, the robot manipulator will stop move. On the other hand, in the case of the first approach (8) the robot will move even if the force applied from the human arm is vanished.

Consequently, it is necessary to decide how the force provided by the human's hand, will be embedded to the system through the control input $u$.

\section{Control Law in the Operational Space}

Assuming that the only input to the robotic manipulator is the wrench (forces and moments) applied by the human's upper limb. This wrench, it is feasible to be measured by a force/torque sensor attached at the end-effector of the artificial limb, and it is a vector of the form $f_{A} \in \mathbb{R}^{6}$.

Let $f_{d} \in \mathbb{R}^{6}$ denote the desired constant wrench reference. This reference is based on the task which we would like the robot manipulator to execute. More specifically, in case of zero interaction between the artificial and human limb this vector of desired forces is became zero $\left(f_{d}=\emptyset\right)$. When the task is for the artificial limb to assist human's movements by amplifying the force that the human applies, this vector is taking the form $f_{d}=\alpha \cdot f_{A}$, where $\alpha$ is a positive constant called amplification factor. In the third case in which we would like the robot manipulator to apply a specific force profile w.r.t. users intention in order to help an injured person, this vector $f_{d}$ is taking the shape of the predefined force profile.

Therefore, the control law $u$ for the representation of the system in the operational space $(8) /(10)$, is given from the following equation:

$$
u=K_{p} \cdot C_{F} \cdot\left(f_{d}-f_{A}\right)
$$

where $\left(f_{d}-f_{A}\right)$ is the force error input, $C_{F}$ is diagonal matrix which describes the control action in the operational space 
and it represents the compliance of the artificial limb, and $K_{p}$ as defined in previous subsection.

By using (2), (4)/(9) and (11), we have performed a position control, taking the end-effector of the robotic manipulator to the desired position and orientation (defined from the measured force applied by the human's hand), according to the dynamics of $(8) /(10)$ specified by the choice of matrices $M_{d}, K_{D}$, and $K_{p}$.

\section{EP CONTROL ANALYSIS}

In this section we will analyze the case of EP control. Before proceeding with our analysis we need the following definition.

Definition 1: Assume that there is a human hand moving free in space. When it collides with an environmental object $\mathscr{O}$, we can measure the produced force $F$ between the hand and the object (Fig. 6). An Equilibrium Position (EP) is defined the point that the hand reach in absence of environmental obstacles and it is related to the produced force in the appearance of an object in this place:

$$
F=K_{h} \cdot E P
$$

where $K_{h}$ is the stiffness matrix of the human hand.

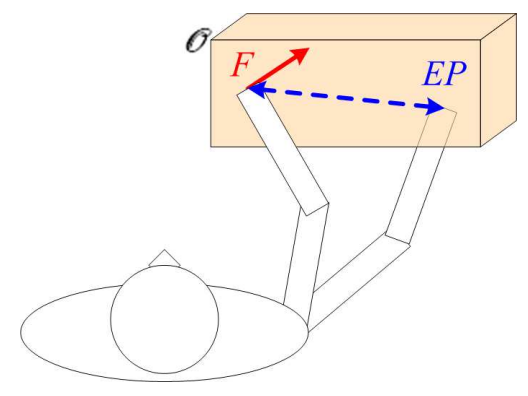

Fig. 6. Equilibrium Position (EP) definition.

In the coupled system of an artificial and a human limb, the human hand understands the robotic manipulator as an environmental object and it pushes against it in order to execute a task. This force appearance is related with its EP. What neuro-scientific models suggest, is to find a way to transfer to the manipulator this human's EP rather than the measured force. In other words it is preferable, according to that models to use the force measurements and to transfer them to the artificial limb as EP, using the following equation:

$$
E P=C_{h} \cdot F
$$

where $C_{h}$ is the mechanical compliance matrix of the hand and it is related with the human's hand stiffness according to the equation $C_{h}=K_{h}^{-1}$. Experiments in human's subjects have been done in order to provide the measurements of human's stiffness matrices, [5].

Consequently, we can now define the control law $u$ for the representation of the system in the operational space $(8) /(10)$, taking into account the EP of the human hand. Therefore, the following equation describes the structure of the control law:

$$
u=K_{p} \cdot C_{h} \cdot\left(f_{d}-f_{A}\right)
$$

where $\left(f_{d}-f_{A}\right)$ is the force error input, $C_{h}$ is the mechanical compliance matrix of the human limb, and $K_{p}, f_{d}$, and $f_{A}$ as defined in previous section.

\section{STABILITY ANALYSIS}

We have the system's representation of the form (8). This system under the control law (11)/(14) takes the following form:

$$
\begin{gathered}
\dot{x}_{1}=x_{2} \\
\dot{x}_{2}=M_{d}^{-1} \cdot\left(-K_{D} \cdot x_{2}-K_{p} \cdot x_{1}+K_{p} \cdot C_{i} \cdot\left(f_{d}-f_{A}\right)\right)
\end{gathered}
$$

where $i=$ " $F$ ", " $h$ " in case of force control and EP control, respectively. Therefore, we have that:

$$
\dot{X}=A \cdot X+B \cdot U
$$

with $X=\left[\begin{array}{c}x_{1} \\ x_{2}\end{array}\right], A=\left[\begin{array}{cc}\emptyset_{m \times m} & I_{m \times m} \\ -M_{d}^{-1} \cdot K_{p} & -M_{d}^{-1} \cdot K_{D}\end{array}\right], B=$ $\left[\begin{array}{c}\emptyset_{m \times m} \\ M_{d}^{-1} \cdot K_{p} \cdot C_{i}\end{array}\right]$ and $U=\left(f_{d}-f_{A}\right)$.

Following the same notation and for the system's representation of the form (10), under the control law (11)/(14), we have that:

$$
\begin{gathered}
\dot{x}_{1}=x_{2} \\
\dot{x}_{2}=M_{d}^{-1} \cdot\left(-K_{D} \cdot x_{2}+K_{p} \cdot C_{i} \cdot\left(f_{d}-f_{A}\right)\right)
\end{gathered}
$$

which is described by the form of (16), but with a difference in $A$ matrix, $A=\left[\begin{array}{cc}\emptyset_{m \times m} & I_{m \times m} \\ \emptyset_{m \times m} & -M_{d}^{-1} \cdot K_{D}\end{array}\right]$.

The stability properties and robustness of the above approach are based on the decision of the contained matrices $M_{d}, K_{D}$, and $K_{p}$, as much as the correctness of the artificial limb's and human's hand compliance matrices.

Also, a very important issue is presented in the case of amplification of human's movements. In this case, a very small movement from the human subject, is sufficient to produce a bigger movement of the artificial limb in order to assist the human. A very large factor of amplification may cause a loss of stability of the coupled system. Thus, it is very significant for a bounded force disturbance to the coupled system, produced from human's intention of movement, to estimate an upper bound of amplification factor for the artificial limb, to keep the coupled system stable. Therefore, in this case when the amplification of human's movement is desired, we have that $B=\left[\begin{array}{c}\emptyset_{m \times m} \\ M_{d}^{-1} \cdot K_{p} \cdot C_{i} \cdot(\alpha-1)\end{array}\right]$ and $U=f_{A}$ of the system (16).

In the direction of resolving the above problems, we have that under the assumptions where $M_{d}$ and $K_{D}$ are the positive definite matrices and $K_{p}$ is the positive semi-definite matrix, it can be shown that a matrix $\Phi$ exists s.t. $\Phi^{T} \cdot M_{d} \cdot \Phi=I$, and $\Phi^{T} \cdot K_{p} \cdot \Phi=\Omega^{2}=\operatorname{diag}\left\{\omega_{j}^{2}\right\}$, such as $\Phi^{T} \cdot K_{D} \cdot \Phi=$ $\Delta=\operatorname{diag}\left\{\zeta_{j}\right\}$, where $\Omega$ is the matrix of the system's natural frequencies, and $\Delta$ is the damping matrix of the system. Also, there exists a positive definite matrix $\mathrm{P}$ s.t. $C^{T}=P \cdot B$. 
The overall transfer matrix $G(s)=C \cdot(s I-A)^{-1} \cdot B$ can be shown to be (strictly) positive real (it is stable and its Nyquist plot is completely contained in the right half complex plane). Moreover, the changes in the uncertain positive coefficients of the above matrices, will not affect the performance of the system, since the above property is robust against those changes. In other words, we can guarantee the robust positive realness, for such a class of uncertain systems, based on the structure of the system equations, [9].

\section{SIMULATION RESULTS}

We have performed simulations in order to test the above control laws. In the simulations we have consider a 2 d.o.f. artificial limb (Fig. 7(a)) which is identical to the 2 d.o.f. of the human arm, for only planar movements (shoulder extension and elbow flexion/extension, Fig. 7(b)).

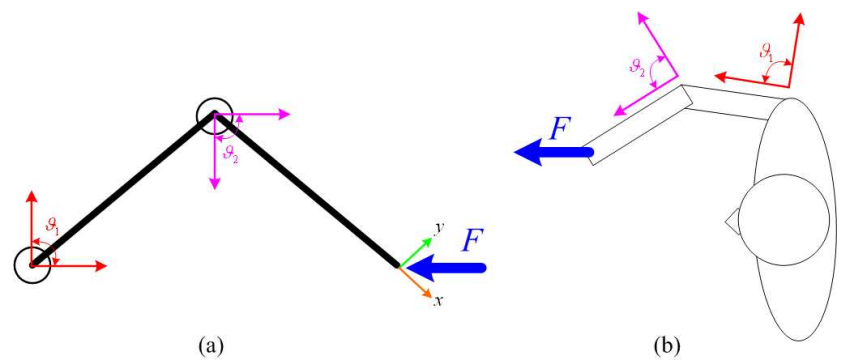

Fig. 7. (a) The 2 d.o.f. set-up of robotic arm. (b) The 2 d.o.f. set-up of human hand (shoulder extension and elbow flexion/extension).

One of the simulated ideas was to make the interaction force between the human hand and the artificial limb to be zero. Thus, it holds that $f_{d}=0$, and we have assumed that the applied force from the human subject $f_{A}$ (measured from the force/torque sensor), is a horizontal force, with the following form:

$$
f_{A}=\left[\begin{array}{llll}
F_{x} & \underbrace{0}_{5} \quad \ldots & 0
\end{array}\right]^{T}
$$

having the profile depicted in Fig. 8(a) (blue line). This force profile simulates the human's intention. At the first step the magnitude of the applied force is increasing. When the robotic arm start its movement in order to compensate this disturbance, the force measurement is constant until the human achieves the desired task and then the force's magnitude is decreasing until this interaction force vanishes.

We have tested the control law for the case of force control with inner position loop, (8) and of force control with inner velocity loop, (10). The simulation results are depicted in Fig. 8(b) and Fig. 8(c), respectively. For the first case when the controller includes a position loop on its structure (red line), we have that the system cannot follow the reference error signal in an acceptable way. More specifically, when the measured horizontal force became zero, the system's velocity is not zero and drives the robot away from the desired position (since there is a position error influence on the control law). At the other hand, in the case of velocity loop (green line), we have that the system can react according to the input signal and stops moving when the force error signal goes to zero.

Furthermore, we have performed simulations in order to achieve an amplification of human's hand produced forces. In this case it holds that $f_{d}=\alpha \cdot f_{A}$, with $\alpha>0$ the amplification factor, where $f_{A}$ is again a horizontal force of the form (18), (blue line of Fig. 9(a), 10(a)). The behavior of $f_{A}$ is the same as described in the previous scenario of the zero interaction force.

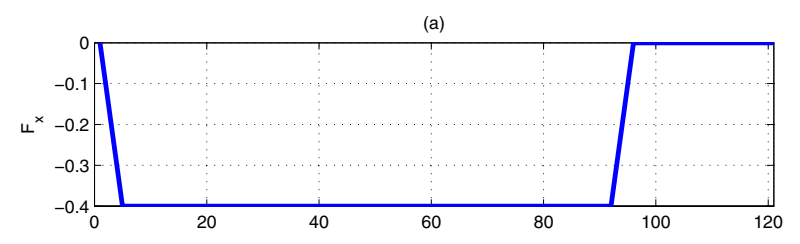

(b)
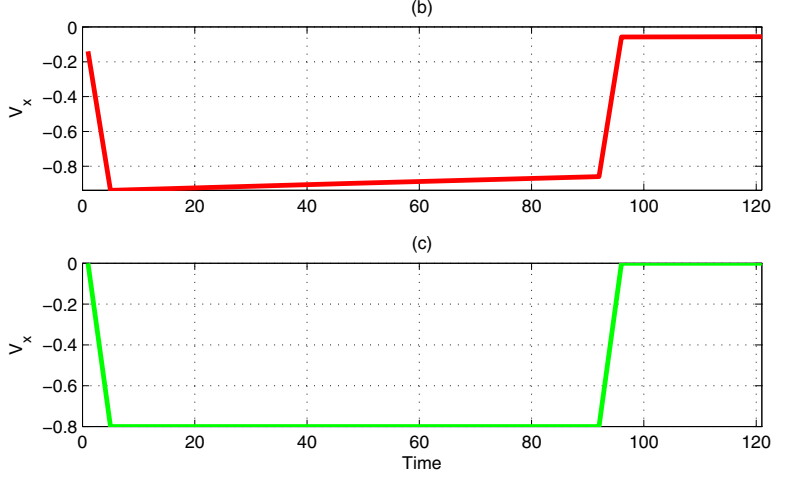

Fig. 8. (a) Horizontal measured force at the end-effector of artificial limb. (b) End-effector's velocity in the horizontal direction, with position loop in the controller structure. (c) End-effector's velocity in the horizontal direction, with velocity loop in the controller structure.

Similarly with the previous performed simulations, we have tested both cases of force control with inner position (red line) / velocity (green line) loop. The simulations have been performed for the case of amplification factor, first, $\alpha=1.3$, Fig. 9 and second, $\alpha=2.8$, Fig. 10 .

In the amplification case's simulation results, we have the same behavior of the tested controllers as with the above simulations (zero interaction force case). Again for the first case, when the controller includes a position loop on its structure, Fig. 9(b) and 10(b), we have that the system cannot follow the reference error signal in an acceptable way. At the other hand, in the case of velocity loop, Fig. 9(c) and 10(c), we have that the system can react according to the input signal and it stops moving, when the force error signal is going to zero.

\section{CONCLUSIONS AND FUTURE WORKS}

\section{A. Conclusions}

We presented a study for a coupled system, between a human upper limb and an artificial arm (robotic manipulator). The main goal of this analysis was to make the robotic arm an assistance of the human hand. The first step was the manipulator to recognize the human's intention and then to help or 
(a)
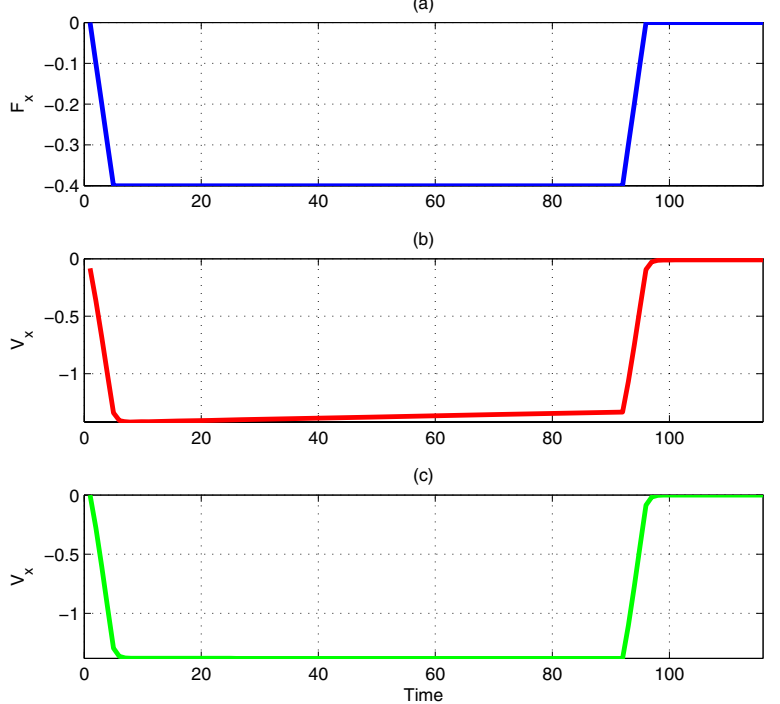

Fig. 9. (a) Horizontal measured force at the end-effector of artificial limb. (b) End-effector's velocity in the horizontal direction, with position loop in the controller structure, and amplification factor $\alpha=1.3$. (c) End-effector's velocity in the horizontal direction, with velocity loop in the controller structure, and amplification factor $\alpha=1.3$.

not resist its movement. We have performed an engineering analysis of how we can control such a coupled system between a robotic/human arm, based on information come from a force/torque sensor attached between the artificial and the real hand. This analysis is according to neuro-scientific results of how humans perceive and reproduce forces, which indicated to explore the fact that human subjects utilize their "sense of effort" rather than the "sense of force" or the "sense of torque", etc.

To this first approach we have tested how we can control the artificial arm in order to implement such validation experiments. The proposed control approach is stable and robust with changes of some parameters.

\section{B. Future Works}

Further research includes the testing of the proposed approaches for a real robotic system, coupled with a human subject. Also, in the future plans is included the case that the coupled system performs a compliant motion, based on environmental constraints. In this scenario a 2-D or 3$\mathrm{D}$ isometric joystick will be attached between the human and artificial limb, act like an environment with a different resistance according to a predefined scenario.

\section{REFERENCES}

[1] Project-FP6-IST-001917-NEUROBOTICS, "The fusion of neuroscience and robotics," in http://www.neurobotics.info, 2004.

[2] J. Houk and W. Rymer, Neural control of muscle length and tension. Vernon B. Brooks Edition. Ch. VIII, Handbook of Physiology - Section 1: The Nervous System - Volume 2: Motor Control, 1981.

[3] L. Jones, "Perception of force and weight: theory and research," PsycholBull, vol. 100, pp. 29-42, 1986.
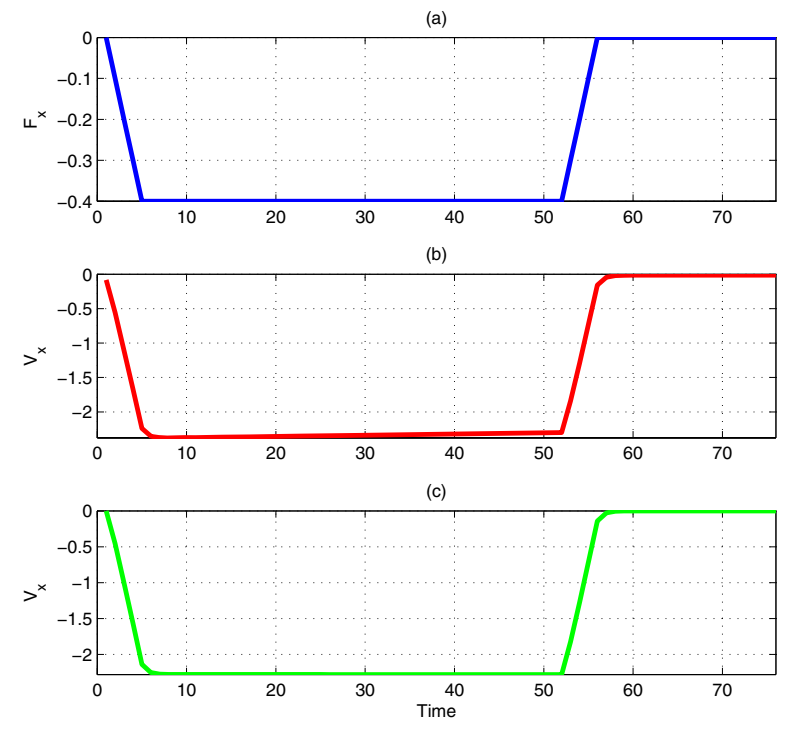

Fig. 10. (a) Horizontal measured force at the end-effector of artificial limb. (b) End-effector's velocity in the horizontal direction, with position loop in the controller structure, and amplification factor $\alpha=2.8$. (c) End-effector's velocity in the horizontal direction, with velocity loop in the controller structure, and amplification factor $\alpha=2.8$.

[4] D. McCloskey, P. Ebeling, and G. Goodwin, "Estimation of weighted and tension and apparent involvement of a "sense of effort", Exp. Neurol., vol. 42, p. 220232, 1974.

[5] D. Toffin, J. McIntyre, J. Droulez, A. Kemeny, and A. Berthoz, "Perception and reproduction of force direction in the horizontal plane," J. Neurophysiology, vol. 90, pp. 3040-3053, 2003.

[6] J. McIntyre and E. Bizzi, "Servo hypotheses for the biological control of movement," J. Motor. Behav., vol. 25, p. 193202, 1993.

[7] A. Feldman, "Once more on the equilibrium-point hypothesis ( $\lambda$ model) for motor control," J. Motor. Behav., vol. 18, p. 1754, 1986.

[8] L. Sciavicco and B. Siciliano, Modeling and Control of Robot Manipulators. McGraw-Hill, 1996.

[9] J. Ackermann, Robust Control Systems with Uncertain Physical Papameters. Springer-Verlag, 1993. 